\title{
Cytokine therapy: clinical and progressive status in cancer
}

\author{
Vemuri Praveen Kumar*, \\ Mamidi Sai Ratna Mounica, \\ Tadikamalla N.L. Swetha, \\ Gogineni V.S.N. Lakshmi, \\ Tullimilli Anusri, Pemmaraju Lakshmi Amulya \\ and Anne Sirisha
}

Department of Biotechnology,

KL University,

Green Fields, Vaddeswaram, Guntur District, 522502, Andhra Pradesh, India

E-mail: vemuripraveen@gmail.com

E-mail: mounica_ratna@yahoo.com

E-mail: tnlswetha@gmail.com

E-mail: vsnlakshmi.gogineni@gmail.com

E-mail: tullimillianusri@gmail.com

E-mail: pemmaraju.amulya@gmail.com

E-mail: sirisha.annes@gmail.com

*Corresponding author

\begin{abstract}
Immunologic approaches to cancer therapy rely on two distinct capabilities of the immune system: targeting the tumour microenvironment by recognising molecules expressed to a greater extent on tumour cells than normal cells and generating immune responses that can kill tumour cells. Immunocytokines, which are fusion proteins composed of a recombinant monoclonal antibody and a cytokine, capitalise on both of these capabilities by combining the ability of tumour-specific antibodies selectively to target tumours with the broad-based immunomodulatory activities of cytokines. This review hypothesises the rationale for development of immunocytokines for cancer and discusses preclinical and clinical data on specific immunocytokines being investigated as potential cancer therapies.
\end{abstract}

Keywords: cytokine; cancer; immunocytokine; monoclonal antibody; aldesleukin; tumour; microenvironment; IL-2; hu14.18-IL-2; huKS-IL-2.

Reference to this paper should be made as follows: Kumar, V.P., Mounica, M.S.R., Swetha, T.N.L., Lakshmi, G.V.S.N., Anusri, T., Amulya, P.L. and Sirisha, A. (2011) 'Cytokine therapy: clinical and progressive status in cancer', Int. J. Immunological Studies, Vol. 1, No. 3, pp.264-271.

Biographical notes: Vemuri Praveen Kumar received his MTech and MSc from the Acharya Nagarjuna University, India. $\mathrm{He}$ is an employee of KL University and presently holding the position of an Assistant Professor. $\mathrm{He}$ is an advisory member for Society of Regenerative Medicine and Tissue Engineering, Bangalore. He has published papers in, among other journals, 
Journal of Biotechnology and Biochemistry, Journal of Experimental Sciences, Journal of Applied Biosciences, as well as a Referee for Journal of Medicine and Medical Sciences and African Journal of Plant Sciences.

Mamidi Sai Ratna Mounica is a student of KL University and presently pursuing her final year graduate programme in biotechnology. She was a class topper and assisted senior scientists for DBT sponsored projects at the Rajiv Gandhi Centre for Biotechnology, Kerala. She also participated in various paper and poster presentations across India.

Tadikamalla N.L. Swetha is a student of KL University and presently pursuing her final year graduate programme in biotechnology. She participated in paper and poster presentations at various universities across India.

Gogineni V.S.N. Lakshmi is a student of KL University and presently pursuing her final year graduate programme in biotechnology. She participated in international conference on biostatistics at the Hyderabad Central University, Andhra Pradesh.

Tullimilli Anusri is a student of KL University and presently pursuing her final year graduate programme in biotechnology. She participated in various poster presentations across Andhra Pradesh.

Pemmaraju Lakshmi Amulya is a student of KL University and presently pursuing her final year graduate programme in biotechnology. She participated in poster presentations and grabbed prizes at various central universities across India as well as selected for Summer Fellowship 2010 and did project at the Indian Institute of Technology, Chennai.

Anne Sirisha is a student of KL University and presently pursuing her final year graduate programme in biotechnology. She participated in paper presentations at various engineering colleges and universities across India.

\section{Introduction}

Cancer is the name given for those diseases in which the body cells become abnormal and divide without control. Cancer cells may invade nearby tissues and they may spread through the bloodstream and lymphatic system to other parts of the body. Its two main characteristics are uncontrolled proliferation of the cells in the human body and ability of these cells to migrate from the original site and spread to distant sites. If the spread is not controlled, cancer can result in death. Cancer, by definition, is a disease of the genes. A gene is a functional unit of DNA, which is the master molecule of the cell. Genes make 'proteins', which are the ultimate workhorses of the cells that allow our bodies to carry out all the processes that permit us to breathe, think, move, etc. Throughout people's lives, the cells in their bodies are growing, dividing, and replacing themselves. Many genes produce proteins that are involved in controlling the processes of cell growth and division. An alteration to the DNA molecule can disrupt the genes and produce faulty proteins. This causes the cell to become abnormal and lose its restraints on growth. The abnormal cell begins to divide uncontrollably and eventually forms a new growth known as a 'tumour' or neoplasm (medical term for cancer meaning 'new growth'). In a healthy 
individual, the immune system can recognise the neoplastic cells and destroy them before they get a chance to divide However, some mutant cells may escape immune detection and survive to become tumours or cancers.

\section{Trends in cancer treatment}

Cancer is a nearly invincible disease that has plagued humankind for centuries. Only in recent decades have doctors found effective ways to treat it and also found better methods for early detection of this devastating disease. But there are still years away from any possible cure for cancer, something that many scientists think is impossible. While early detection is the best form of prevention, there are several techniques that are used to treat cancer. These techniques include surgery, radiation therapy, chemotherapy, hormone therapy and immunotherapy.

\subsection{Surgery}

Surgery is the oldest and most widely used treatment available for cancer patients. If a growth is found early, there is a good chance to remove an entire tumour before it spreads. Surgery is rarely used as a stand-alone treatment. Usually, it is combined with radiation therapy and/or chemotherapy.

\subsection{Radiation therapy}

The specific part of the body containing cancerous growth is exposed to radiation energy to attack reproducing cancer cells, to shrink a tumour so that it can be removed through surgery, or to prevent tumour growth following surgery. However, radiation affects normal cells along with the cancer cells which lead to several unpleasant side effects, including fatigue, dryness and peeling of skin, nausea and vomiting.

\subsection{Chemotherapy}

The treatment of cancer through drugs is an effective treatment method for fighting cancerous cells that have spread to other parts of the body and that cannot be treated with any other method. Similar to radiation therapy, chemotherapy also can affect normal cells, causing the same kinds of side effects.

Two more recent treatments for cancer are hormone therapy and immunotherapy (Cheng et al., 2000). Hormone therapy involves anything that deals with manipulating the body's hormones to treat the cancer, including administering hormones and drugs. It may also involve removal of hormone glands to kill cancer cells or prevent further cancerous growth. Immunotherapy also manipulates the body's normal functions. During immunotherapy, patients are given medication to stimulate the body's immune system to fight cancerous cells. Many different compounds of biological origin that are used in the immune response can now be made in the laboratory; these are interferon's, Interleukin-2 (IL-2), Monoclonal antibodies. Interferon-alpha and IL-2 might act by boosting the immune response to help the body kill off cancer cells. 


\section{Immunocytokines: rationale and hypothesised mechanism of action}

Immunocytokines combine the elements of two established modalities of immunotherapy: recombinant monoclonal antibody therapy and cytokine therapy.

\subsection{Monoclonal antibodies for cancer therapy}

The administration of recombinant monoclonal antibodies for cancer is based on the premise that tumour-specific antibodies can recognise and target cancer cells and, once in the tumour microenvironment, can inhibit tumour growth or induces tumour regression via mechanisms including antibody-dependent cell-mediated cytotoxicity (ADCC) and complement-dependent cytotoxicity (CDC) (Lode et al., 1998). The anti-CD20 monoclonal antibody (Maloney et al., 1994) rituximab, for example, binds to the B-cell antigen CD20 (Reff et al., 1994) expressed on B-cell lymphomas to kill cancer cells via ADCC and induction of apoptosis (antibody (Maloney et al., 1997). The monoclonal antibody trastuzumab binds to the receptor for the growth factor HER-2, which is over-expressed in up to $30 \%$ of human breast cancers, to block the proliferative effects of this growth factor on breast cancer cells (Harries and Smith, 2002). Monoclonal antibodies have also been conjugated to tumour-killing substances such as radioisotopes, drugs, or toxins (Goldenberg et al., 1995). Although monoclonal antibody therapy has yielded positive results in some human cancers, it has several limitations. Shortcomings include poor penetration of solid masses with resulting impaired distribution of antibody at the tumour, heterogeneity of antigen expression leading to suboptimal monoclonal binding to tumour, and limited direct activation of tumour cell destruction.

\subsection{Cytokines for cancer therapy}

Cytokines such as interferons and interleukins are administered for cancer because of their broad-based immunostimulatory effects including generation of tumour-reactive lymphocytes. IL-2 or aldesleukin, which is indicated for the treatment of adults with metastatic renal cell carcinoma and melanoma, is the most studied cytokine. IL-2 dose-dependently activates cellular immunity and causes release of other immune-boosting cytokines in vivo. Systemic cytokine therapy is generally limited by rapid degradation and elimination of the cytokine, the inability to achieve optimal concentrations in the tumour microenvironment (Mocellin et al., 2001), and dose dependent toxicity, including life-threatening side effects such as vascular leak syndrome and orthostatic hypotension (Baluna and Vitetta, 1997). Cytokine gene therapy, in which a cytokine gene (such as that for IL-2) is introduced into tumour cells, is being explored to overcome some of these limitations of systemic cytokine administration. However, cytokine gene therapy is technologically challenging and resource-intensive.

\section{Rationale for immunocytokines}

As fusion proteins combining monoclonal antibodies with cytokines, immunocytokines were developed to improve upon the benefit: risk ratios of monoclonal antibodies and cytokines alone or as combination therapy. Immunocytokines are hypothesised to confer two possible advantages over currently available immunotherapies (Lode et al., 1998). 
First, by providing both antibody effector activities and a cytokine co signal for the generation of cytotoxic cellular immunity, immunocytokines may amplify antitumour immune responses relative to those obtained with current immunotherapies. Second, by targeting delivery of cytokines to the tumour microenvironment, immunocytokines deliver biologically active concentrations of cytokines at lower and less toxic doses than are required by systemic cytokine therapy. Data from in vitro studies and in vivo tumour models have borne out these hypotheses by showing that immunocytokines stimulate tumour-suppressing immune responses and that in vivo administration of immunocytokines causes a greater antitumour effect than administration of a mixture of an equivalent dose of antibody and cytokine. Immunocytokines also appear to prolong cytokine biological activity relative to that of systemically administered cytokines.

\section{Hypothesised mechanism of action of immunocytokines}

The mechanisms of immunocytokine-mediated antitumour activity, like those of the antitumour activity of monoclonal antibodies or cytokines administered alone, are not fully understood but appear to be multifactorial (Gillies et al., 1992; Reisfeld and Gillies, 1996). Once bound to its specific antigen on the tumour cell surface, the antibody portion of the immunocytokine is hypothesised to mediate both ADCC - which involves antibody activation of effector cells (Sondel and Hank, 2001) such as macrophages, granulocytes, monocytes, and some natural killer (NK) cells - and CDC, which involves activation of the complement cascade (Figure 1). The cytokine portion of the immunocytokine is hypothesised to mediate a range of immunomodulatory responses in the tumour microenvironment by activating cells of the immune system through their cytokine receptors. The cytokine portion of the immunocytokine also provides a costimulatory signal for enhancing both NK-cell- and T-cell-mediated antitumour responses.

Figure 1 Hypothesised mechanisms of antitumour action of immunocytokines

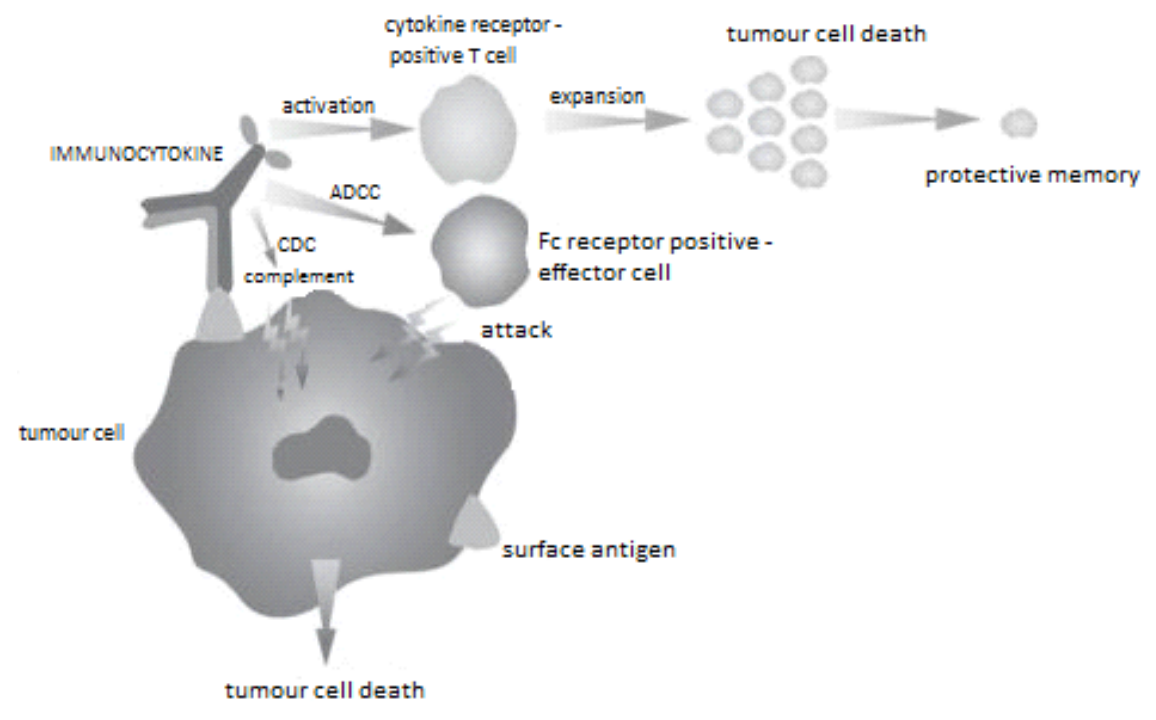




\section{Immunocytokines in development}

Several immunocytokines are being explored for potential use in human cancers, and two (hu14.18-IL-2 and huKS-IL-2) have been studied in human cancer with promising results to date. Besides these IL-2-based immunocytokines, which have been thoroughly assessed in preclinical models and are undergoing evaluation in the clinic studies, IL-12- and granulocytemacrophage colony-stimulating factor-based (GM-CSF-based) immunocytokines are being assessed in preclinical tumour models.

\subsection{Anti GD-IL2 immunocytokine}

The anti-GD2 immunocytokine hu14.18-IL-2 (Parry, 2001) (EMD 273063) is currently in phase I clinical trials. This immunocytokine consists of recombinant human IL-2 and a humanised monoclonal antibody directed against human GD2, which is a disialoganglioside antigen expressed by tumours of neuroectodermal origin including malignant melanoma, neuroblastoma, some sarcomas, and some small-cell lung carcinomas. Each immunocytokine molecule is composed of two cytokine molecules fused to each of the two heavy chains of a monoclonal antibody via a peptide linkage at the carboxyl terminus (Figure 2).

Figure 2 Immunocytokine hu14.18-IL-2

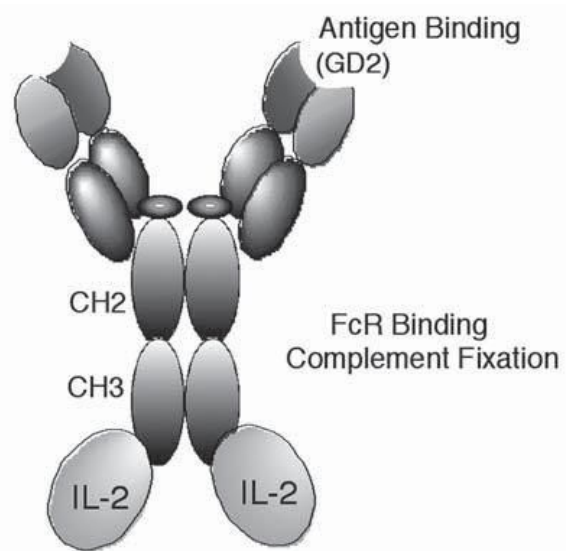

\section{Biological activity}

Preclinical studies with the anti-GD2-IL-2 fusion protein were conducted with a chimeric form (i.e., ch14.18-IL-2) as well as the humanised form that is EMD 273063 (i.e., hu14.18-IL-2). Early studies focused on establishing the biological functionality of the immunocytokine and on determining whether the fused protein retained full antibody and cytokine functions. That ch14.18-IL-2 retains its functionality as an antibody was confirmed by the finding that the immunocytokine and ch14.18 had comparable GD2-binding patterns in direct binding assays with human neuroblastoma cells (Sabzevari et al., 1994). Dissociation constants (Kd) for the immunocytokine and ch14.18 were $24 \mathrm{nM}$ and $18 \mathrm{nM}$, respectively. A similar pattern of results was observed in 
separate studies with hu14.18-IL-2. That ch14.18-IL-2 retained cytokine properties was confirmed by the finding that the fusion of ch14.18 with IL-2 did not reduce IL-2 activity relative to that of commercially available IL-2 in a standard proliferation assay involving either mouse or human T-cell lines. Furthermore, the ability of a melanomaspecific T-cell line derived from tumour-infiltrating lymphocytes (TIL) to kill autologous GD2-positive melanoma tumour cells was enhanced when target cells were precoated with ch14.18-IL-2. This effect of the ch14.18-IL-2 exceeded that observed when these TILs were cultured with autologous tumour cells in higher concentrations of free IL-2 (Gillies et al., 1992). In another study, human lymphocyte-activated killer cells created by incubating human peripheral blood mononuclear cells with recombinant human IL-2 showed enhanced ADCC when pretreated with ch14.18-IL-2 immunocytokine $(10 \mathrm{mg} / \mathrm{mL}$ containing 30,000 IU of recombinant human IL-2) compared with administration of either ch14.18 alone $(10 \mathrm{mg} / \mathrm{mL})$ or a mixture of ch14.18 $(10 \mathrm{mg} / \mathrm{mL})$ and 30,000 IU recombinant human IL-2. Analyses of serum from mice injected intravenously with ch14.18-IL-2 show that the half-life of the immunocytokine was approximately $4 \mathrm{~h}$ (Kendra et al., 1999). This value is shorter than the half-life of the ch14.18 monoclonal antibody but substantially longer than the half life of soluble IL-2.

\section{Conclusions}

The preclinical and early clinical data on immunocytokines for cancer therapy suggest that this new therapeutic modality has promise for improving outcomes for patients with cancer. As administration of immunocytokines poses no apparent risk of cross-resistance with conventional therapeutic modalities including chemotherapeutics, radiotherapy, and surgery, combination therapy appears to be feasible and should be explored. Preclinical data suggest that the greatest long-term tumour protection by immunocytokines is obtained in animals with minimal tumour burdens. Thus, regimens that include immunocytokines merit development and testing in experimental animals and patients achieving clinical remission via conventional treatments but likely to experience recurrence. Combination therapy involving chemotherapeutic agents and immunocytokines, in particular, may be a fruitful approach given the preclinical work demonstrating synergy of immunocytokines and standard chemotherapies with respect to antitumour effects. Besides combination therapy involving immunocytokines and standard cancer treatments, combinations involving immunocytokines and other immunomodulatory approaches such as gene therapy may constitute a promising avenue for additional research and have begun to be assessed in preclinical models.

\section{References}

Baluna, R. and Vitetta, E.S. (1997) 'Vascular leak syndrome: a side effect of immunotherapy', Immunopharmacology, Vol. 37, pp.117-132.

Cheng, J.D., Rieger, P.T., Von Mehren, M. et al. (2000) 'Recent advances in immunotherapy and monoclonal antibody treatment of cancer', Semin. Oncol. Nurs., Vol. 16, Suppl. 1, pp.2-12.

Gillies, S.D., Reilly, E.B., Lo, K-M. et al. (1992) 'Antibody-targeted interleukin-2 stimulates T cell killing of autologous tumor cells', Proc. Natl. Acad. Sci., USA, Vol. 89, pp.1428-1432.

Goldenberg, D.M., Larson, S.M., Reisfeld, R.A. et al. (1995) 'Targeting cancer with radiolabeled antibodies', Immunol. Today, Vol. 16, pp.261-264. 
Harries, M. and Smith, I. (2002) 'The development and clinical use of trastuzumab', Endocr. Relat. Cancer, Vol. 9, pp.75-85.

Kendra, K., Gan, J., Ricci, M. et al. (1999) 'Pharmacokinetics and stability of the 14.18-IL-2 fusion protein in mice', Cancer Immunol. Immunother., Vol. 48, pp.219-229.

Lode, H.N., Xiang, R., Becker, J.C. et al. (1998) 'Immunocytokines: a promising approach to cancer immunotherapy', Pharmacol. Ther., Vol. 80, pp.277-292.

Maloney, D.G., Grillo-Lopez, A.J., White, C.A. et al. (1997) 'IDEC-C2B8 (rituximab) anti-CD20 monoclonal anti-body therapy in patients with relapsed low-grade non-Hodgkin's lymphoma', Blood, Vol. 90, pp.2188-2195.

Maloney, D.G., Liles, T.M., Czerwinski, D.K. et al. (1994) 'Phase I clinical trial using escalating single-dose infusion of chimeric anti-CD20 monoclonal antibody in patients with recurrent B cell lymphoma', Blood, Vol. 84, pp.2457-2466.

Mocellin, S., Wang, E. and Marincola, F.M. (2001) 'Cytokines and immune response in the tumor microenvironment', J. Immunother, Vol. 24, pp.392-407.

Parry, J.L. (2001) 'hu14.18-IL-2 investigator's brochure', Version 2. EMD Pharmaceuticals, October.

Reff, M.E., Carner, K., Chambers, K.S. et al. (1994) 'Depletion of B cells in vivo by a chimeric mouse human monoclonal antibody to CD20', Blood, Vol. 83, pp.434-445.

Reisfeld, R.A. and Gillies, S.D. (1996) 'Antibody-interleukin-2 fusion proteins: a new approach to cancer therapy', J. Clin. Lab. Anal., Vol. 10, pp.160-166.

Sabzevari, H., Gillies, S.D., Mueller, B.M. et al. (1994) 'A recombinant antibody-interleukin-2 fusion protein suppresses growth of hepatic human neuroblastoma metastases in severe combined immunodeficiency mice', Proc. Natl. Acad. Sci., USA, Vol. 91, pp.9626-9630.

Sondel, P.M. and Hank, J.A. (2001) 'Antibody-directed, effector cellmediated tumor destruction', Hem. Oncol. Clinical North Am., Vol. 15, pp.703-721. 